\title{
Constraints on Cosmic Strings from the LIGO-Virgo Gravitational-Wave Detectors
}

J. Aasi, ${ }^{1}$ J. Abadie, ${ }^{1}$ B. P. Abbott, ${ }^{1}$ R. Abbott, ${ }^{1}$ T. Abbott,${ }^{2}$ M. R. Abernathy, ${ }^{1}$ T. Accadia, ${ }^{3}$ F. Acernese,${ }^{4,5}$ C. Adams,${ }^{6}$ T. Adams ${ }^{7}$ R. X. Adhikari, ${ }^{1}$ C. Affeldt, ${ }^{8}$ M. Agathos, ${ }^{9}$ N. Aggarwal, ${ }^{10}$ O. D. Aguiar,${ }^{11}$ P. Ajith,,${ }^{1}$ B. Allen,${ }^{8,12,13}$ A. Allocca, ${ }^{14,15}$ E. Amador Ceron, ${ }^{12}$ D. Amariutei ${ }_{1}^{16}$ R. A. Anderson, ${ }^{1}$ S. B. Anderson, ${ }^{1}$ W. G. Anderson, ${ }^{12}$ K. Arai, ${ }^{1}$ M. C. Araya, ${ }^{1}$ C. Arceneaux,${ }^{17}$ J. Areeda,${ }^{18}$ S. Ast,${ }^{13}$ S. M. Aston,${ }^{6}$ P. Astone,${ }^{19}$ P. Aufmuth, ${ }^{13}$ C. Aulbert, ${ }^{8}$ L. Austin, ${ }^{1}$ B. E. Aylott ${ }^{20}$ S. Babak, ${ }^{21}$ P. T. Baker, ${ }^{22}$ G. Ballardin, ${ }^{23}$ S. W. Ballmer,${ }^{24}$ J. C. Barayoga, ${ }^{1}$ D. Barker, ${ }^{25}$ S. H. Barnum, ${ }^{10}$ F. Barone,${ }^{4,5}$ B. Barr, ${ }^{26}$ L. Barsotti, ${ }^{10}$ M. Barsuglia, ${ }^{27}$ M. A. Barton, ${ }^{25}$ I. Bartos, ${ }^{28}$ R. Bassiri, ${ }^{29,26}$ A. Basti, ${ }^{14,30}$ J. Batch, ${ }^{25}$ J. Bauchrowitz, ${ }^{8}$ Th. S. Bauer, ${ }^{9}$ M. Bebronne, ${ }^{3}$ B. Behnke, ${ }^{21}$ M. Bejger, ${ }^{31}$ M. G. Beker, ${ }^{9}$ A. S. Bell, ${ }^{26}$ C. Bell, ${ }^{26}$

I. Belopolski, ${ }^{28}$ G. Bergmann, ${ }^{8}$ J. M. Berliner, ${ }^{25}$ D. Bersanetti, ${ }^{32,33}$ A. Bertolini, ${ }^{9}$ D. Bessis, ${ }^{34}$ J. Betzwieser, ${ }^{6}$

P. T. Beyersdorf, ${ }^{35}$ T. Bhadbhade, ${ }^{29}$ I. A. Bilenko, ${ }^{36}$ G. Billingsley, ${ }^{1}$ J. Birch,${ }^{6}$ M. Bitossi, ${ }^{14}$ M. A. Bizouard, ${ }^{37}$ E. Black, ${ }^{1}$ J. K. Blackburn, ${ }^{1}$ L. Blackburn, ${ }^{38}$ D. Blair, ${ }^{39}$ M. Blom, ${ }^{9}$ O. Bock, ${ }^{8}$ T. P. Bodiya,,${ }^{10}$ M. Boer, ${ }^{40}$ C. Bogan, ${ }^{8}$ C. Bond, ${ }^{20}$

F. Bondu ${ }^{41}$ L. Bonelli, ${ }^{14,30}$ R. Bonnand,${ }^{42}$ R. Bork, ${ }^{1}$ M. Born, ${ }^{8}$ V. Boschi,${ }^{14}$ S. Bose, ${ }^{43}$ L. Bosi,${ }^{44}$ J. Bowers, ${ }^{2}$ C. Bradaschia, ${ }^{14}$ P. R. Brady, ${ }^{12}$ V. B. Braginsky, ${ }^{36}$ M. Branchesi, ${ }^{45,46}$ C. A. Brannen, ${ }^{43}$ J. E. Brau, ${ }^{47}$ J. Breyer, ${ }^{8}$ T. Briant, ${ }^{48}$ D. O. Bridges, ${ }^{6}$ A. Brillet, ${ }^{40}$ M. Brinkmann, ${ }^{8}$ V. Brisson, ${ }^{37}$ M. Britzger, ${ }^{8}$ A. F. Brooks, ${ }^{1}$ D. A. Brown, ${ }^{24}$ D. D. Brown, ${ }^{20}$ F. Brückner, ${ }^{20}$ T. Bulik ${ }^{49}$ H. J. Bulten,${ }^{9,50}$ A. Buonanno, ${ }^{51}$ D. Buskulic, ${ }^{3}$ C. Buy,${ }^{27}$ R. L. Byer,${ }^{29}$ L. Cadonati, ${ }^{52}$ G. Cagnoli, ${ }^{42}$ J. Calderón Bustillo, ${ }^{53}$ E. Calloni, ${ }^{4,54}$ J. B. Camp, ${ }^{38}$ P. Campsie, ${ }^{26}$ K. C. Cannon, ${ }^{55}$ B. Canuel, ${ }^{23}$ J. Cao, ${ }^{56}$ C. D. Capano ${ }^{51}$ F. Carbognani, ${ }^{23}$ L. Carbone, ${ }^{20}$ S. Caride, ${ }^{57}$ A. Castiglia, ${ }^{58}$ S. Caudill, ${ }^{12}$ M. Cavaglià, ${ }^{17}$ F. Cavalier, ${ }^{37}$ R. Cavalieri, ${ }^{23}$ G. Cella, ${ }^{14}$ C. Cepeda, ${ }^{1}$ E. Cesarini,${ }^{59}$ R. Chakraborty, ${ }^{1}$ T. Chalermsongsak, ${ }^{1}$ S. Chao,${ }^{60}$ P. Charlton, ${ }^{61}$

E. Chassande-Mottin, ${ }^{27}$ X. Chen,${ }^{39}$ Y. Chen, ${ }^{62}$ A. Chincarini, ${ }^{32}$ A. Chiummo, ${ }^{23}$ H. S. Cho, ${ }^{63}$ J. Chow, ${ }^{64}$ N. Christensen, ${ }^{65}$ Q. Chu, ${ }^{39}$ S. S. Y. Chua ${ }^{64}$ S. Chung, ${ }^{39}$ G. Ciani, ${ }^{16}$ F. Clara, ${ }^{25}$ D. E. Clark, ${ }^{29}$ J. A. Clark, ${ }^{52}$ F. Cleva, ${ }^{40}$ E. Coccia,${ }^{66,67}$ P.-F. Cohadon, ${ }^{48}$ A. Colla, ${ }^{19,68}$ M. Colombini, ${ }^{44}$ M. Constancio, Jr., ${ }^{11}$ A. Conte, ${ }^{19,68}$ R. Conte, ${ }^{69}$ D. Cook, ${ }^{25}$ T. R. Corbitt, ${ }^{2}$ M. Cordier, ${ }^{35}$ N. Cornish, ${ }^{22}$ A. Corsi,${ }^{70}$ C. A. Costa, ${ }^{11}$ M. W. Coughlin, ${ }^{71}$ J.-P. Coulon, ${ }^{40}$ S. Countryman, ${ }^{28}$ P. Couvares, ${ }^{24}$ D. M. Coward, ${ }^{39}$ M. Cowart, ${ }^{6}$ D. C. Coyne, ${ }^{1}$ K. Craig, ${ }^{26}$ J. D. E. Creighton, ${ }^{12}$ T. D. Creighton, ${ }^{34}$ S. G. Crowder, ${ }^{72}$ A. Cumming, ${ }^{26}$ L. Cunningham, ${ }^{26}$ E. Cuoco, ${ }^{23}$ K. Dahl, ${ }^{8}$ T. Dal Canton, ${ }^{8}$ M. Damjanic, ${ }^{8}$ S. L. Danilishin, ${ }^{39}$ S. D' Antonio, ${ }^{59}$ K. Danzmann, ${ }^{8,13}$ V. Dattilo, ${ }^{23}$ B. Daudert, ${ }^{1}$ H. Daveloza, ${ }^{34}$ M. Davier,${ }^{37}$ G. S. Davies, ${ }^{26}$ E. J. Daw ${ }^{73}$ R. Day, ${ }^{23}$ T. Dayanga ${ }^{43}$ R. De Rosa, ${ }^{4,54}$ G. Debreczeni, ${ }^{74}$ J. Degallaix,${ }^{42}$ W. Del Pozzo, ${ }^{9}$ E. Deleeuw, ${ }^{16}$ S. Deléglise, ${ }^{48}$ T. Denker, ${ }^{8}$ T. Dent, ${ }^{8}$ H. Dereli, ${ }^{40}$ V. Dergachev, ${ }^{1}$ R. DeRosa,${ }^{2}$ R. DeSalvo, ${ }^{69}$ S. Dhurandhar, ${ }^{75}$ L. Di Fiore, ${ }^{4}$ A. Di Lieto, ${ }^{14,30}$ I. Di Palma, ${ }^{8}$ A. Di Virgilio, ${ }^{14}$ M. Díaz, ${ }^{34}$ A. Dietz, ${ }^{17}$ K. Dmitry, ${ }^{36}$ F. Donovan, ${ }^{10}$ K. L. Dooley, ${ }^{8}$ S. Doravari, ${ }^{6}$ M. Drago, ${ }^{76,77}$ R. W. P. Drever, ${ }^{78}$ J. C. Driggers, ${ }^{1}$ Z. Du, ${ }^{56}$ J.-C. Dumas, ${ }^{39}$ S. Dwyer, ${ }^{25}$ T. Eberle, ${ }^{8}$ M. Edwards, ${ }^{7}$ A. Effler, ${ }^{2}$ P. Ehrens, ${ }^{1}$ J. Eichholz, ${ }^{16}$ S. S. Eikenberry, ${ }^{16}$ G. Endrőczi, ${ }^{74}$ R. Essick, ${ }^{10}$ T. Etzel, ${ }^{1}$ K. Evans, ${ }^{26}$ M. Evans, ${ }^{10}$ T. Evans, ${ }^{6}$

M. Factourovich, ${ }^{28}$ V. Fafone, ${ }^{59,67}$ S. Fairhurst, ${ }^{7}$ Q. Fang, ${ }^{39}$ S. Farinon, ${ }^{32}$ B. Farr, ${ }^{79}$ W. Farr, ${ }^{79}$ M. Favata, ${ }^{80}$ D. Fazi, ${ }^{79}$ H. Fehrmann, ${ }^{8}$ D. Feldbaum, ${ }^{16,6}$ I. Ferrante, ${ }^{14,30}$ F. Ferrini, ${ }^{23}$ F. Fidecaro, ${ }^{14,30}$ L. S. Finn, ${ }^{81}$ I. Fiori, ${ }^{23}$ R. Fisher, ${ }^{24}$

R. Flaminio, ${ }^{42}$ E. Foley, ${ }^{18}$ S. Foley, ${ }^{10}$ E. Forsi ${ }^{6}{ }^{2}$. Fotopoulos, ${ }^{1}$ J.-D. Fournier, ${ }^{40}$ S. Franco, ${ }^{37}$ S. Frasca, ${ }^{19,68}$ F. Frasconi,${ }^{14}$ M. Frede ${ }^{8}$ M. Frei, ${ }^{58}$ Z. Frei ${ }^{82}$ A. Freise,${ }^{20}$ R. Frey,${ }^{47}$ T. T. Fricke, ${ }^{8}$ P. Fritschel,${ }^{10}$ V. V. Frolov, ${ }^{6}$ M.-K. Fujimoto, ${ }^{83}$ P. Fulda, ${ }^{16}$ M. Fyffe, ${ }^{6}$ J. Gair, ${ }^{71}$ L. Gammaitoni, ${ }^{44,84}$ J. Garcia, ${ }^{25}$ F. Garufi, ${ }^{4,54}$ N. Gehrels, ${ }^{38}$ G. Gemme, ${ }^{32}$ E. Genin, ${ }^{23}$ A. Gennai, ${ }^{14}$ L. Gergely, ${ }^{82}$ S. Ghosh,${ }^{43}$ J. A. Giaime, ${ }^{2,6}$ S. Giampanis, ${ }^{12}$ K. D. Giardina, ${ }^{6}$ A. Giazotto, ${ }^{14}$ S. Gil-Casanova, ${ }^{53}$ C. Gill, ${ }^{26}$ J. Gleason, ${ }^{16}$ E. Goetz, ${ }^{8}$ R. Goetz, ${ }^{16}$ L. Gondan, ${ }^{82}$ G. González, ${ }^{2}$ N. Gordon,${ }^{26}$ M. L. Gorodetsky, ${ }^{36}$ S. Gossan, ${ }^{62}$ S. Goßler, ${ }^{8}$ R. Gouaty, ${ }^{3}$ C. Graef, ${ }^{8}$ P. B. Graff, ${ }^{38}$ M. Granata, ${ }^{42}$ A. Grant, ${ }^{26}$ S. Gras, ${ }^{10}$ C. Gray, ${ }^{25}$ R. J. S. Greenhalgh, ${ }^{85}$ A. M. Gretarsson, ${ }^{86}$ C. Griffo, ${ }^{18}$ P. Groot,${ }^{87}$ H. Grote,${ }^{8}$ K. Grover, ${ }^{20}$ S. Grunewald, ${ }^{21}$ G. M. Guidi, ${ }^{45,46}$ C. Guido, ${ }^{6}$ K. E. Gushwa, ${ }^{1}$ E. K. Gustafson, ${ }^{1}$ R. Gustafson, ${ }^{57}$ B. Hall,${ }^{43}$ E. Hall, ${ }^{1}$ D. Hammer, ${ }^{12}$ G. Hammond,${ }^{26}$ M. Hanke, ${ }^{8}$ J. Hanks ${ }^{25}$ C. Hanna,${ }^{88}$ J. Hanson, ${ }^{6}$ J. Harms, ${ }^{1}$ G. M. Harry ${ }^{89}$ I. W. Harry ${ }^{24}$ E. D. Harstad ${ }^{47}$ M. T. Hartman, ${ }^{16}$ K. Haughian,${ }^{26}$ K. Hayama ${ }^{83}$ J. Heefner, ${ }^{1,}$ A. Heidmann, ${ }^{48}$ M. Heintze, ${ }^{16,6}$ H. Heitmann, ${ }^{40}$ P. Hello, ${ }^{37}$ G. Hemming, ${ }^{23}$ M. Hendry, ${ }^{26}$ I. S. Heng, ${ }^{26}$ A. W. Heptonstall, ${ }^{1}$ M. Heurs ${ }^{8}$ S. Hild,${ }^{26}$ D. Hoak, ${ }^{52}$ K. A. Hodge, ${ }^{1}$ K. Holt,${ }^{6}$ M. Holtrop, ${ }^{90}$ T. Hong, ${ }^{62}$ S. Hooper, ${ }^{39}$ T. Horrom, ${ }^{91}$ D. J. Hosken, ${ }^{92}$ J. Hough ${ }^{26}$ E. J. Howell, ${ }^{39}$ Y. Hu, ${ }^{26}$ Z. Hua ${ }^{56}$ V. Huang, ${ }^{60}$ E. A. Huerta, ${ }^{24}$ B. Hughey, ${ }^{86}$ S. Husa ${ }^{53}$ S. H. Huttner ${ }^{26}$ M. Huynh, ${ }^{12}$ T. Huynh-Dinh, ${ }^{6}$ J. Iafrate, ${ }^{2}$ D. R. Ingram, ${ }^{25}$ R. Inta, ${ }^{64}$ T. Isogai, ${ }^{10}$ A. Ivanov, ${ }^{1}$ B. R. Iyer, ${ }^{93}$ K. Izumi, ${ }^{25}$ M. Jacobson, ${ }^{1}$ E. James, ${ }^{1}$ H. Jang, ${ }^{94}$ Y. J. Jang ${ }^{79}$ P. Jaranowski, ${ }^{95}$ F. Jiménez-Forteza, ${ }^{53}$ W. W. Johnson, ${ }^{2}$ D. Jones, ${ }^{25}$ D. I. Jones, ${ }^{96}$ R. Jones, ${ }^{26}$ R. J. G. Jonker, ${ }^{9}$ L. Ju, ${ }^{39}$ Haris K., ${ }^{97}$ P. Kalmus, ${ }^{1}$ V. Kalogera, ${ }^{79}$ S. Kandhasamy, ${ }^{72}$ G. Kang, ${ }^{44}$ J. B. Kanner, ${ }^{38}$ M. Kasprzack, ${ }^{23,37}$ R. Kasturi, ${ }^{98}$ E. Katsavounidis, ${ }^{10}$ W. Katzman, ${ }^{6}$ H. Kaufer, ${ }^{13}$ K. Kaufman, ${ }^{62}$ K. Kawabe, ${ }^{25}$ S. Kawamura ${ }^{83}$ F. Kawazoe, ${ }^{8}$ F. Kéfélian, ${ }^{40}$ D. Keitel, ${ }^{8}$ D. B. Kelley, ${ }^{24}$ W. Kells, ${ }^{1}$ 
D. G. Keppel, ${ }^{8}$ A. Khalaidovski, ${ }^{8}$ F. Y. Khalili, ${ }^{36}$ E. A. Khazanov, ${ }^{99}$ B. K. Kim,${ }^{94}$ C. Kim, ${ }^{100,94}$ K. Kim, ${ }^{101}$ N. Kim, ${ }^{29}$ W. Kim, ${ }^{92}$ Y.-M. Kim, ${ }^{63}$ E. J. King ${ }^{92}$ P. J. King, ${ }^{1}$ D. L. Kinzel, ${ }^{6}$ J. S. Kissel, ${ }^{10}$ S. Klimenko, ${ }^{16}$ J. Kline, ${ }^{12}$ S. Koehlenbeck, ${ }^{8}$ K. Kokeyama, ${ }^{2}$ V. Kondrashov, ${ }^{1}$ S. Koranda, ${ }^{12}$ W. Z. Korth, ${ }^{1}$ I. Kowalska, ${ }^{49}$ D. Kozak, ${ }^{1}$ A. Kremin, ${ }^{72}$ V. Kringel, ${ }^{8}$ A. Królak, ${ }^{102,103}$ C. Kucharczyk, ${ }^{29}$ S. Kudla, ${ }^{2}$ G. Kuehn, ${ }^{8}$ A. Kumar, ${ }^{104}$ P. Kumar, ${ }^{24}$ R. Kumar ${ }^{26}$ R. Kurdyumov, ${ }^{29}$ P. Kwee, ${ }^{10}$ M. Landry, ${ }^{25}$ B. Lantz, ${ }^{29}$ S. Larson, ${ }^{105}$ P. D. Lasky, ${ }^{106}$ C. Lawrie, ${ }^{26}$ A. Lazzarini, ${ }^{1}$ A. Le Roux, ${ }^{6}$ P. Leaci, ${ }^{21}$ E. O. Lebigot, ${ }^{56}$ C.-H. Lee ${ }^{63}$ H. K. Lee, ${ }^{101}$ H. M. Lee, ${ }^{100}$ J. Lee, ${ }^{10}$ J. Lee ${ }^{18}$ M. Leonardi, ${ }^{76,77}$ J. R. Leong, ${ }^{8}$ N. Leroy, ${ }^{37}$ N. Letendre, ${ }^{3}$ B. Levine, ${ }^{25}$ J. B. Lewis, ${ }^{1}$ V. Lhuillier, ${ }^{25}$ T. G. F. Li, ${ }^{9}$ A. C. Lin ${ }^{29}$ T. B. Littenberg, ${ }^{79}$ V. Litvine, ${ }^{1}$ F. Liu, ${ }^{107}$ H. Liu, ${ }^{7}$ Y. Liu, ${ }^{56}$ Z. Liu, ${ }^{16}$ D. Lloyd, ${ }^{1}$ N. A. Lockerbie, ${ }^{108}$ V. Lockett, ${ }^{18}$ D. Lodhia, ${ }^{20}$ K. Loew, ${ }^{86}$ J. Logue, ${ }^{26}$

A. L. Lombardi, ${ }^{52}$ M. Lorenzini, ${ }^{59}$ V. Loriette, ${ }^{109}$ M. Lormand, ${ }^{6}$ G. Losurdo, ${ }^{45}$ J. Lough, ${ }^{24}$ J. Luan, ${ }^{62}$ M. J. Lubinski, ${ }^{25}$ H. Lück, ${ }^{8,13}$ A. P. Lundgren, ${ }^{8}$ J. Macarthur, ${ }^{26}$ E. Macdonald, ${ }^{7}$ B. Machenschalk, ${ }^{8}$ M. MacInnis, ${ }^{10}$ D. M. Macleod, ${ }^{7}$ F. Magana-Sandoval, ${ }^{18}$ M. Mageswaran, ${ }^{1}$ K. Mailand, ${ }^{1}$ E. Majorana,${ }^{19}$ I. Maksimovic ${ }^{109}$ V. Malvezzi,${ }^{59}$ N. Man, ${ }^{40}$ G. M. Manca, ${ }^{8}$ I. Mandel,${ }^{20}$ V. Mandic,${ }^{72}$ V. Mangano, ${ }^{19,68}$ M. Mantovani,${ }^{14}$ F. Marchesoni,${ }^{44,110}$ F. Marion, ${ }^{3}$ S. Márka, ${ }^{28}$ Z. Márka, ${ }^{28}$ A. Markosyan, ${ }^{29}$ E. Maros, ${ }^{1}$ J. Marque, ${ }^{23}$ F. Martelli, ${ }^{45,46}$ I. W. Martin, ${ }^{26}$ R. M. Martin, ${ }^{16}$ L. Martinelli, ${ }^{40}$ D. Martynov, ${ }^{1}$ J. N. Marx, ${ }^{1}$ K. Mason, ${ }^{10}$ A. Masserot,${ }^{3}$ T. J. Massinger, ${ }^{24}$ F. Matichard,${ }^{10}$ L. Matone, ${ }^{28}$ R. A. Matzner, ${ }^{111}$ N. Mavalvala, ${ }^{10}$ G. May, ${ }^{2}$ N. Mazumder, ${ }^{97}$ G. Mazzolo, ${ }^{8}$ R. McCarthy,${ }^{25}$ D. E. McClelland, ${ }^{64}$ S. C. McGuire, ${ }^{12}$ G. McIntyre, ${ }^{1}$ J. McIver, ${ }^{52}$ D. Meacher, ${ }^{40}$ G. D. Meadors,${ }^{57}$ M. Mehmet ${ }^{8}$ J. Meidam, ${ }^{9}$ T. Meier, ${ }^{13}$ A. Melatos, ${ }^{106}$ G. Mendell, ${ }^{25}$ R. A. Mercer, ${ }^{12}$ S. Meshkov, ${ }^{1}$ C. Messenger, ${ }^{26}$ M. S. Meyer, ${ }^{6}$ H. Miao, ${ }^{62}$ C. Michel, ${ }^{42}$ E. E. Mikhailov, ${ }^{91}$ L. Milano, ${ }^{4,54}$ J. Miller ${ }^{64}$ Y. Minenkov, ${ }^{59}$ C. M. F. Mingarelli, ${ }^{20}$ S. Mitra ${ }^{75}$ V. P. Mitrofanov, ${ }^{36}$ G. Mitselmakher ${ }^{16}$ R. Mittleman, ${ }^{10}$ B. Moe, ${ }^{12}$ M. Mohan, ${ }^{23}$ S. R. P. Mohapatra, ${ }^{24,58}$ F. Mokler, ${ }^{8}$ D. Moraru, ${ }^{25}$ G. Moreno, ${ }^{25}$ N. Morgado, ${ }^{42}$ T. Mori ${ }^{83}$ S. R. Morriss ${ }^{34}$ K. Mossavi, ${ }^{8}$ B. Mours, ${ }^{3}$ C. M. Mow-Lowry, ${ }^{8}$ C. L. Mueller, ${ }^{16}$ G. Mueller ${ }^{16}$ S. Mukherjee,${ }^{34}$ A. Mullavey, ${ }^{2}$ J. Munch, ${ }^{92}$ D. Murphy, ${ }^{28}$ P. G. Murray, ${ }^{26}$ A. Mytidis, ${ }^{16}$ M. F. Nagy, ${ }^{74}$ D. Nanda Kumar, ${ }^{16}$ I. Nardecchia,,${ }^{19,68}$ T. Nash, ${ }^{1}$ L. Naticchioni, ${ }^{19,68}$ R. Nayak, ${ }^{113}$ V. Necula, ${ }^{16}$ G. Nelemans, ${ }^{87,9}$ I. Neri ${ }^{44,84}$ M. Neri, ${ }^{32,33}$ G. Newton, ${ }^{26}$ T. Nguyen,${ }^{64}$ E. Nishida, ${ }^{83}$ A. Nishizawa, ${ }^{83}$ A. Nitz, ${ }^{24}$ F. Nocera, ${ }^{23}$ D. Nolting, ${ }^{6}$ M. E. Normandin, ${ }^{34}$ L. K. Nuttall, ${ }^{7}$ E. Ochsner, ${ }^{12}$ J. O'Dell, ${ }^{85}$ E. Oelker, ${ }^{10}$ G. H. Ogin, ${ }^{1}$ J. J. Oh, ${ }^{114}$ S. H. Oh, ${ }^{114}$ F. Ohme, ${ }^{7}$ P. Oppermann, ${ }^{8}$ B. O'Reilly, ${ }^{6}$ W. Ortega Larcher, ${ }^{34}$ R. O’Shaughnessy, ${ }^{12}$ C. Osthelder, ${ }^{1}$ C. D. Ott, ${ }^{62}$ D. J. Ottaway, ${ }^{92}$ R. S. Ottens, ${ }^{16}$ J. Ou, ${ }^{60}$ H. Overmier, ${ }^{6}$ B. J. Owen, ${ }^{81}$ C. Padilla, ${ }^{18}$ A. Pai,${ }^{97}$ C. Palomba, ${ }^{19}$ Y. Pan, ${ }^{51}$ C. Pankow, ${ }^{12}$ F. Paoletti, ${ }^{14,23}$ R. Paoletti, ${ }^{14,15}$ M. A. Papa,${ }^{21,12}$ H. Paris,${ }^{25}$ A. Pasqualetti, ${ }^{23}$ R. Passaquieti, ${ }^{14,30}$ D. Passuello, ${ }^{14}$ M. Pedraza, ${ }^{1}$ P. Peiris, ${ }^{58}$ S. Penn, ${ }^{98}$ A. Perreca, ${ }^{24}$ M. Phelps, ${ }^{1}$ M. Pichot, ${ }^{40}$ M. Pickenpack,${ }^{8}$ F. Piergiovanni, ${ }^{45,46}$ V. Pierro, ${ }^{69}$ L. Pinard, ${ }^{42}$ B. Pindor,${ }^{106}$ I. M. Pinto, ${ }^{69}$ M. Pitkin, ${ }^{26}$ J. Poeld, ${ }^{8}$ R. Poggiani ${ }^{14,30}$ V. Poole,${ }^{43}$ C. Poux,${ }^{1}$ V. Predoi,${ }^{7}$ T. Prestegard, ${ }^{72}$ L. R. Price, ${ }^{1}$ M. Prijatelj, ${ }^{8}$ M. Principe, ${ }^{69}$ S. Privitera, ${ }^{1}$ R. Prix,${ }^{8}$ G. A. Prodi, ${ }^{76,77}$ L. Prokhorov, ${ }^{36}$ O. Puncken, ${ }^{34}$ M. Punturo, ${ }^{44}$ P. Puppo, ${ }^{19}$ V. Quetschke, ${ }^{34}$ E. Quintero, ${ }^{1}$ R. Quitzow-James, ${ }^{47}$ F. J. Raab, ${ }^{25}$ D. S. Rabeling, ${ }^{9,50}$ I. Rácz, ${ }^{74}$ H. Radkins, ${ }^{25}$ P. Raffai, ${ }^{28,82}$ S. Raja, ${ }^{115}$ G. Rajalakshmi, ${ }^{116}$ M. Rakhmanov, ${ }^{34}$ C. Ramet, ${ }^{6}$ P. Rapagnani, ${ }^{19,68}$ V. Raymond, ${ }^{1}$ V. Re,${ }^{59,67}$ C. M. Reed ${ }^{25}$ T. Reed, ${ }^{117}$ T. Regimbau, ${ }^{40}$ S. Reid ${ }^{118}$ D. H. Reitze, ${ }^{1,16}$ F. Ricci, ${ }^{19,68}$ R. Riesen, ${ }^{6}$ K. Riles ${ }^{57}$ N. A. Robertson, ${ }^{1,26}$ F. Robinet, ${ }^{37}$ A. Rocchi, ${ }^{59}$ S. Roddy, ${ }^{6}$ C. Rodriguez, ${ }^{79}$ M. Rodruck, ${ }^{25}$ C. Roever, ${ }^{8}$ L. Rolland, ${ }^{3}$ J. G. Rollins, ${ }^{1}$ R. Romano, ${ }^{4,5}$ G. Romanov, ${ }^{91}$ J. H. Romie, ${ }^{6}$ D. Rosińska, ${ }^{31,119}$ S. Rowan, ${ }^{26}$ A. Rüdiger, ${ }^{8}$ P. Ruggi, ${ }^{23}$ K. Ryan, ${ }^{25}$ F. Salemi, ${ }^{8}$ L. Sammut, ${ }^{106}$ V. Sandberg, ${ }^{25}$ J. Sanders,${ }^{57}$ V. Sannibale, ${ }^{1}$ I. Santiago-Prieto, ${ }^{26}$ E. Saracco, ${ }^{42}$ B. Sassolas, ${ }^{42}$ B. S. Sathyaprakash, ${ }^{7}$ P. R. Saulson, ${ }^{24}$ R. Savage, ${ }^{25}$ R. Schilling, ${ }^{8}$ R. Schnabel, ${ }^{8,13}$ R. M. S. Schofield, ${ }^{47}$ E. Schreiber, ${ }^{8}$ D. Schuette, ${ }^{8}$ B. Schulz, ${ }^{8}$ B. F. Schutz,${ }^{21,7}$ P. Schwinberg, ${ }^{25}$ J. Scott, ${ }^{26}$ S. M. Scott, ${ }^{64}$ F. Seifert, ${ }^{1}$ D. Sellers, ${ }^{6}$ A. S. Sengupta, ${ }^{120}$ D. Sentenac, ${ }^{23}$ A. Sergeev, ${ }^{99}$ D. Shaddock, ${ }^{64}$ S. Shah, ${ }^{87,9}$ M. S. Shahriar, ${ }^{79}$ M. Shaltev, ${ }^{8}$ B. Shapiro, ${ }^{29}$ P. Shawhan, ${ }^{51}$ D. H. Shoemaker, ${ }^{10}$ T. L. Sidery, ${ }^{20}$ K. Siellez, ${ }^{40}$ X. Siemens, ${ }^{12}$ D. Sigg, ${ }^{25}$ D. Simakov, ${ }^{8}$ A. Singer,${ }^{1}$ L. Singer, ${ }^{1}$ A. M. Sintes,${ }^{53}$ G. R. Skelton, ${ }^{12}$

B. J. J. Slagmolen, ${ }^{64}$ J. Slutsky, ${ }^{8}$ J. R. Smith, ${ }^{18}$ M. R. Smith, ${ }^{1}$ R. J. E. Smith, ${ }^{20}$ N. D. Smith-Lefebvre, ${ }^{1}$ K. Soden, ${ }^{12}$ E. J. Son, ${ }^{114}$ B. Sorazu, ${ }^{26}$ T. Souradeep,${ }^{75}$ L. Sperandio, ${ }^{59,67}$ A. Staley, ${ }^{28}$ E. Steinert, ${ }^{25}$ J. Steinlechner, ${ }^{8}$ S. Steinlechner, ${ }^{8}$ S. Steplewski, ${ }^{43}$ D. Stevens, ${ }^{79}$ A. Stochino, ${ }^{64}$ R. Stone, ${ }^{34}$ K. A. Strain, ${ }^{26}$ N. Straniero, ${ }^{42}$ S. Strigin, ${ }^{36}$ A. S. Stroeer, ${ }^{34}$ R. Sturani, ${ }^{45,46}$ A. L. Stuver, ${ }^{6}$ T. Z. Summerscales, ${ }^{121}$ S. Susmithan, ${ }^{39}$ P. J. Sutton, ${ }^{7}$ B. Swinkels, ${ }^{23}$ G. Szeifert, ${ }^{82}$ M. Tacca, ${ }^{27}$ D. Talukder, ${ }^{47}$ L. Tang ${ }^{34}$ D. B. Tanner, ${ }^{16}$ S. P. Tarabrin, ${ }^{8}$ R. Taylor, ${ }^{1}$ A. P. M. ter Braack,,${ }^{9}$ M. P. Thirugnanasambandam, ${ }^{1}$ M. Thomas, ${ }^{6}$ P. Thomas, ${ }^{25}$ K. A. Thorne, ${ }^{6}$ K. S. Thorne, ${ }^{62}$ E. Thrane, ${ }^{1}$ V. Tiwari, ${ }^{16}$ K. V. Tokmakov,${ }^{108}$ C. Tomlinson ${ }^{73}$ A. Toncelli, ${ }^{14,30}$ M. Tonelli, ${ }^{14,30}$ O. Torre, ${ }^{14,15}$ C. V. Torres,${ }^{34}$ C. I. Torrie, ${ }^{1,26}$ F. Travasso, ${ }^{44,84}$ G. Traylor, ${ }^{6}$ M. Tse, ${ }^{28}$

D. Ugolini, ${ }^{122}$ C. S. Unnikrishnan, ${ }^{116}$ H. Vahlbruch, ${ }^{13}$ G. Vajente, ${ }^{14,30}$ M. Vallisneri, ${ }^{62}$ J. F. J. van den Brand,,${ }^{9,50}$ C. Van Den Broeck, ${ }^{9}$ S. van der Putten, ${ }^{9}$ M. V. van der Sluys, ${ }^{87,9}$ J. van Heijningen, ${ }^{9}$ A. A. van Veggel, ${ }^{26}$ S. Vass, ${ }^{1}$ M. Vasúth, ${ }^{74}$ R. Vaulin, ${ }^{10}$ A. Vecchio, ${ }^{20}$ G. Vedovato, ${ }^{123}$ J. Veitch, ${ }^{9}$ P. J. Veitch, ${ }^{92}$ K. Venkateswara, ${ }^{124}$ D. Verkindt ${ }^{3}$ S. Verma, ${ }^{39}$ F. Vetrano, ${ }^{45,46}$ A. Viceré, ${ }^{45,46}$ R. Vincent-Finley, ${ }^{112}$ J.-Y. Vinet, ${ }^{40}$ S. Vitale, ${ }^{10,9}$ B. Vlcek, ${ }^{12}$ T. Vo, ${ }^{25}$ H. Vocca, ${ }^{44,84}$ 
C. Vorvick, ${ }^{25}$ W. D. Vousden, ${ }^{20}$ D. Vrinceanu, ${ }^{34}$ S. P. Vyachanin, ${ }^{36}$ A. Wade,${ }^{64}$ L. Wade, ${ }^{12}$ M. Wade, ${ }^{12}$ S. J. Waldman, ${ }^{10}$ M. Walker, ${ }^{2}$ L. Wallace, ${ }^{1}$ Y. Wan, ${ }^{56}$ J. Wang ${ }^{60}$ M. Wang, ${ }^{20}$ X. Wang, ${ }^{56}$ A. Wanner, ${ }^{8}$ R. L. Ward, ${ }^{64}$ M. Was, ${ }^{8}$ B. Weaver ${ }^{25}$ L.-W. Wei, ${ }^{40}$ M. Weinert, ${ }^{8}$ A. J. Weinstein, ${ }^{1}$ R. Weiss, ${ }^{10}$ T. Welborn, ${ }^{6}$ L. Wen, ${ }^{39}$ P. Wessels, ${ }^{8}$ M. West,${ }^{24}$ T. Westphal, ${ }^{8}$ K. Wette, ${ }^{8}$ J. T. Whelan, ${ }^{58}$ S. E. Whitcomb,${ }^{1,39}$ D. J. White, ${ }^{73}$ B. F. Whiting, ${ }^{16}$ S. Wibowo, ${ }^{12}$ K. Wiesner, ${ }^{8}$ C. Wilkinson, ${ }^{25}$ L. Williams,${ }^{16}$ R. Williams, ${ }^{1}$ T. Williams, ${ }^{125}$ J. L. Willis, ${ }^{126}$ B. Willke, ${ }^{8,13}$ M. Wimmer, ${ }^{8}$ L. Winkelmann, ${ }^{8}$ W. Winkler, ${ }^{8}$ C. C. Wipf,${ }^{10}$ H. Wittel, ${ }^{8}$ G. Woan,${ }^{26}$ J. Worden,${ }^{25}$ J. Yablon, ${ }^{79}$ I. Yakushin, ${ }^{6}$ H. Yamamoto, ${ }^{1}$ C. C. Yancey, ${ }^{51}$ H. Yang,${ }^{62}$ D. Yeaton-Massey, ${ }_{1}$ S. Yoshida, ${ }^{125}$ H. Yum, ${ }^{79}$ M. Yvert, ${ }^{3}$ A. Zadrożny, ${ }^{103}$ M. Zanolin, ${ }^{86}$ J.-P. Zendri, ${ }^{123}$ F. Zhang, ${ }^{10}$ L. Zhang, ${ }^{1}$ C. Zhao, ${ }^{39}$ H. Zhu, ${ }^{81}$ X. J. Zhu, ${ }^{39}$ N. Zotov, ${ }^{117,}{ }^{1}$ M. E. Zucker, ${ }^{10}$ and J. Zweizig ${ }^{1}$

(LIGO Scientific Collaboration and Virgo Collaboration)

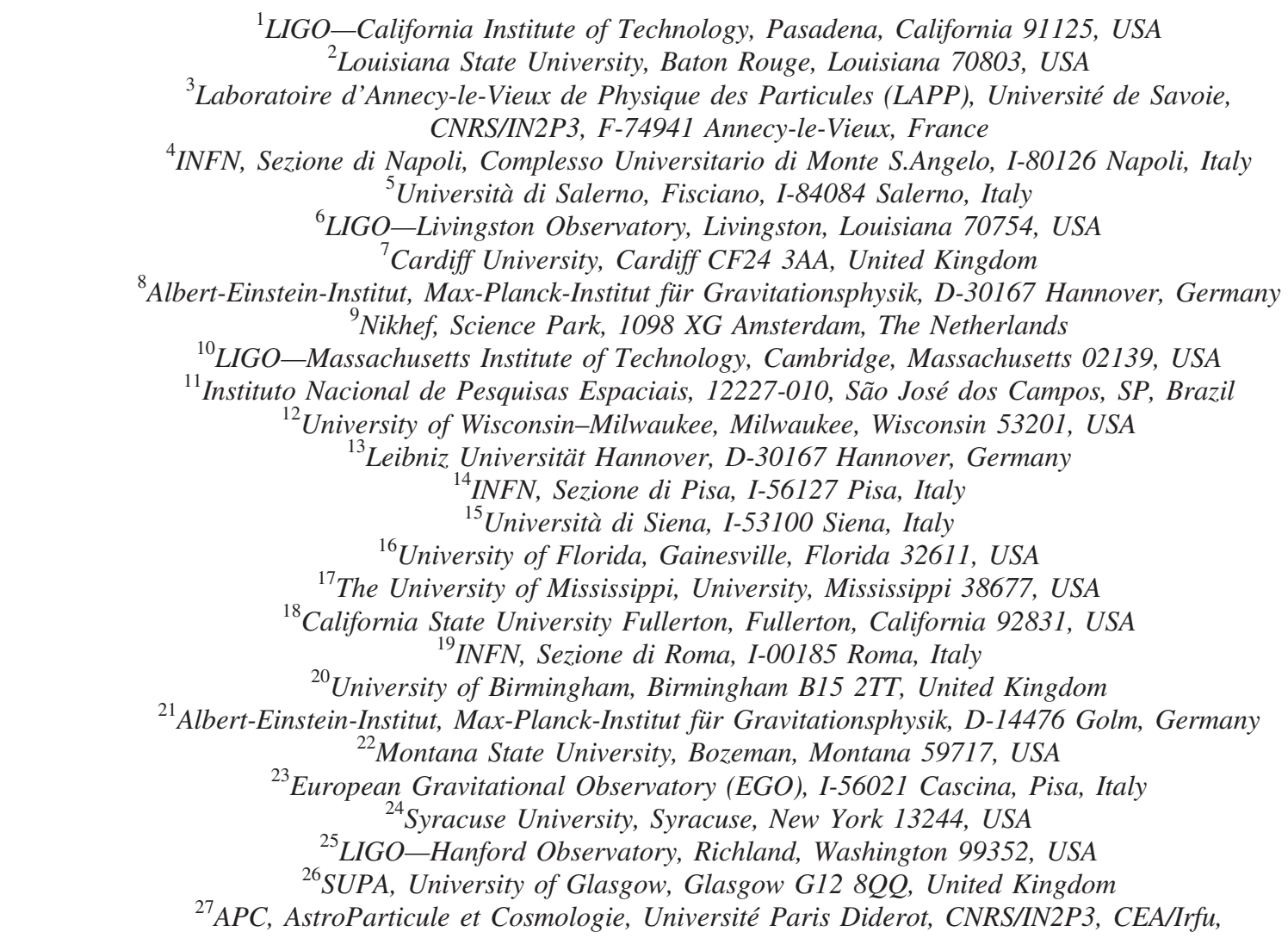

Observatoire de Paris, Sorbonne Paris Cité, 10, rue Alice Domon et Léonie Duquet, F-75205 Paris Cedex 13, France

${ }^{28}$ Columbia University, New York, New York 10027, USA

${ }^{29}$ Stanford University, Stanford, California 94305, USA

${ }^{30}$ Università di Pisa, I-56127 Pisa, Italy

${ }^{31}$ CAMK-PAN, 00-716 Warsaw, Poland

${ }^{32}$ INFN, Sezione di Genova, I-16146 Genova, Italy

${ }^{33}$ Università degli Studi di Genova, I-16146 Genova, Italy

${ }^{34}$ The University of Texas at Brownsville, Brownsville, Texas 78520, USA

${ }^{35}$ San Jose State University, San Jose, California 95192, USA

${ }^{36}$ Moscow State University, Moscow 119992, Russia

${ }^{37}$ LAL, Université Paris-Sud, IN2P3/CNRS, F-91898 Orsay, France

${ }^{38}$ NASA/Goddard Space Flight Center, Greenbelt, Maryland 20771, USA

${ }^{39}$ University of Western Australia, Crawley, WA 6009, Australia

${ }^{40}$ Université Nice-Sophia-Antipolis, CNRS, Observatoire de la Côte d'Azur, F-06304 Nice, France

${ }^{41}$ Institut de Physique de Rennes, CNRS, Université de Rennes 1, F-35042 Rennes, France

${ }^{42}$ Laboratoire des Matériaux Avancés (LMA), IN2P3/CNRS, Université de Lyon, F-69622 Villeurbanne, Lyon, France

${ }^{43}$ Washington State University, Pullman, Washington 99164, USA

${ }^{44}$ INFN, Sezione di Perugia, I-06123 Perugia, Italy

${ }^{45}$ INFN, Sezione di Firenze, I-50019 Sesto Fiorentino, Firenze, Italy

${ }^{46}$ Università degli Studi di Urbino "Carlo Bo", I-61029 Urbino, Italy 


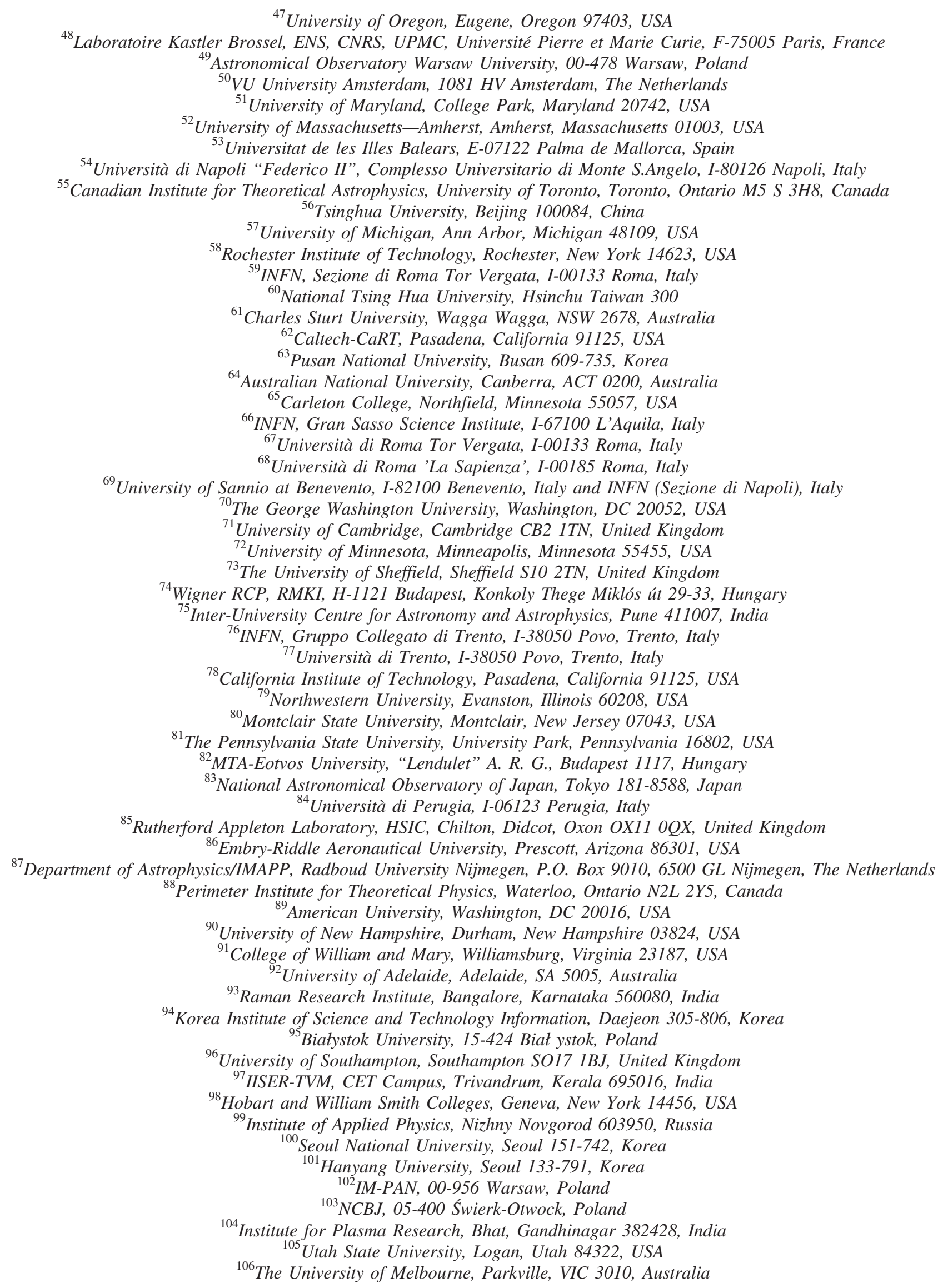




\author{
${ }^{107}$ University of Brussels, Brussels 1050 Belgium \\ ${ }^{108}$ SUPA, University of Strathclyde, Glasgow G1 IXQ, United Kingdom \\ ${ }^{109}$ ESPCI, CNRS, F-75005 Paris, France \\ ${ }^{110}$ Università di Camerino, Dipartimento di Fisica, I-62032 Camerino, Italy \\ ${ }^{111}$ The University of Texas at Austin, Austin, Texas 78712, USA \\ ${ }^{112}$ Southern University and A\&M College, Baton Rouge, Louisiana 70813, USA \\ ${ }^{113}$ IISER-Kolkata, Mohanpur, West Bengal 741252, India \\ ${ }^{114}$ National Institute for Mathematical Sciences, Daejeon 305-390, Korea \\ ${ }^{115}$ RRCAT, Indore, MP 452013, India \\ ${ }^{116}$ Tata Institute for Fundamental Research, Mumbai 400005, India \\ ${ }^{117}$ Louisiana Tech University, Ruston, Louisiana 71272, USA \\ ${ }^{118}$ SUPA, University of the West of Scotland, Paisley PA1 2BE, United Kingdom \\ ${ }^{119}$ Institute of Astronomy, 65-265 Zielona Góra, Poland \\ ${ }^{120}$ Indian Institute of Technology, Gandhinagar, Ahmedabad, Gujarat 382424, India \\ ${ }^{121}$ Andrews University, Berrien Springs, Michigan 49104, USA \\ ${ }^{122}$ Trinity University, San Antonio, Texas 78212, USA \\ ${ }^{123}$ INFN, Sezione di Padova, I-35131 Padova, Italy \\ ${ }^{124}$ University of Washington, Seattle, Washington 98195, USA \\ ${ }^{125}$ Southeastern Louisiana University, Hammond, Louisiana 70402, USA \\ ${ }^{126}$ Abilene Christian University, Abilene, Texas 79699, USA
}

(Received 25 October 2013; published 4 April 2014)

Cosmic strings can give rise to a large variety of interesting astrophysical phenomena. Among them, powerful bursts of gravitational waves (GWs) produced by cusps are a promising observational signature. In this Letter we present a search for GWs from cosmic string cusps in data collected by the LIGO and Virgo gravitational wave detectors between 2005 and 2010, with over 625 days of live time. We find no evidence of GW signals from cosmic strings. From this result, we derive new constraints on cosmic string parameters, which complement and improve existing limits from previous searches for a stochastic background of GWs from cosmic microwave background measurements and pulsar timing data. In particular, if the size of loops is given by the gravitational backreaction scale, we place upper limits on the string tension $G \mu$ below $10^{-8}$ in some regions of the cosmic string parameter space.

Introduction.-A cosmic network of strings may form as a result of phase transitions in the early Universe [1]. When a U(1) symmetry is broken in multiple causally disconnected spacetime regions, one-dimensional topological defects, i.e., strings, are expected to form [2]. For a long time, cosmic strings were considered candidate sources for structure formation in the early Universe [3]. Cosmic microwave background (CMB) experiments, however, have shown that cosmic strings can only contribute up to a few percent of the overall anisotropies observed [4-8]. More recently it was realized that strings can also be produced within the framework of string theory inspired cosmological models and grow to cosmic scales [9-13]. Cosmic strings produced in string theory motivated models (dubbed cosmic superstrings) have received much attention since they could provide observational signatures of string theory $[14,15]$.

Observational constraints on cosmic string models are often given as bounds on the string tension $G \mu(c=1)$, where $G$ is Newton's constant and $\mu$ the mass per unit length. Such constraints have been derived from direct searches for line discontinuities in the CMB temperature maps [16-18] and from simulations of string-sourced CMB anisotropies $[4-7,19,20]$. These analyses, based on various assumptions about the string network, set upper limits on $G \mu$ in the range of $10^{-7}-10^{-6}$. The recent results from the Planck mission [8] constrain $G \mu$ to be lower than $1.5 \times 10^{-7}$ and $3.2 \times 10^{-7}$ for Nambu-Goto and AbelianHiggs strings, respectively.

A promising way of detecting the presence of cosmic strings and superstrings is the gravitational wave (GW) emission from loops [21,22]. When two string segments meet, they exchange partners or intercommute with a probability $p$. For superstrings, the reconnection probability can be less than unity $\left(10^{-4}<p<1\right.$ [23]) while field theory simulations show that topological strings will essentially always reconnect. This is partly due to the fact that fundamental strings interact probabilistically. Furthermore, superstring models have extra spatial dimensions so that even though two strings may meet in three dimensions, they miss each other in the extra dimensions. When a string intercommutes with itself, a closed loop breaks off. The loop oscillates, radiates gravitationally, and decays. Cosmic string loops can form cusps, points along the string with large Lorentz boosts, that produce powerful bursts of gravitational radiation [24]. This Letter reports on the search for such GW transient signatures 
from cosmic strings in data from the LIGO and Virgo gravitational wave detectors.

The GW emission by cusps depends on the loop size, which is often written as a fraction of the horizon at the time of formation $l=\alpha t$, where $t$ is the cosmic time. Early simulations such as Ref. [25] suggested that the size of loops is set by gravitational backreaction and $\alpha \leq \Gamma G \mu$, where $\Gamma \sim 50$ [2]. More recent simulations favor cosmic string networks where the size of loops is dictated by the large scale dynamics of the network, in which case $\alpha \lesssim 1$ $[26,27]$. In this case loops are large and they are long lived because their gravitational decay takes many Hubble times. This Letter only reports constraints for the small loop regime, since the large loop scenario is already well constrained by pulsar data [7,28]. We parametrize $\alpha=\varepsilon \Gamma G \mu$ with $\varepsilon<1$ following the convention of Ref. [21].

Constraints on $G \mu$ were previously derived from observational limits on the stochastic GW background expected from the incoherent superposition of GW emission from cosmic string loops [22]. The tightest limits were obtained, for the case of large loops, by pulsar timing experiments $[7,22,28]$ with $G \mu \lesssim 10^{-9}$ for $p=1$ and $G \mu \lesssim 10^{-12}$ for $p<10^{-2}$ with $\alpha \sim 0.1$. Constraints from pulsar timing experiments were also derived for small loops [22] and are included in Fig. 2. LIGO complemented these results with observational limits from its own search for GW stochastic background in the very small loop region [29]: $G \mu \lesssim 3 \times$ $10^{-8}$ for $p<10^{-3}$ and for $\varepsilon \sim 10^{-11}$. Additional bounds on the GW background can be indirectly derived from CMB [30] data and big-bang nucleosynthesis constraints [31]. At the epochs of last scattering and big-bang nucleosynthesis, the energy density of the GW background must be sufficiently small so as not to distort the CMB fluctuations or affect the abundances of primordial elements. The CMB bound is shown in Fig. 2 and, until the present publication, offered the best limit on $G \mu$ for intermediate values $\varepsilon$. However this indirect limit only applies to gravitational waves generated prior to decoupling, while LIGO and pulsar timing data are also sensitive to later production of GWs.

GW bursts from cosmic string cusps.-This Letter presents a different approach to constrain cosmic string parameters, with a targeted search for transient cusp signatures in LIGO and Virgo data. This approach was previously tested in Ref. [32] over a short period of about 2 weeks of live time with detectors less sensitive by about a factor of 2. For this work we have analyzed all available LIGO and Virgo data collected between 2005 and 2010, at design sensitivity. Moreover, the search pipeline includes new postprocessing techniques, described in this Letter, offering the tightest observational constraints achievable from first generation ground-based GW interferometers.

The possibility of direct detection of GW bursts from cosmic string cusps was first suggested in 2000 by Berezinsky et al. [33]. Shortly after, Damour and Vilenkin showed that the stochastic GW background generated by oscillating loops is strongly non-Gaussian [24]. Occasional sharp bursts of GWs produced by cusps are expected to stand out above the stochastic background [21,24,34]. Damour and Vilenkin predict that the GW burst signal produced by cusps is linearly polarized and the expected waveform in the frequency domain is $h_{\text {cusp }}(f)=A f^{-4 / 3}$ with an exponential decay that sets on at frequency $f_{h}$. The signal amplitude $A$ is determined by the string tension, the loop size, and the propagation distance. The high frequency cutoff $f_{h}$ is determined by the size of the loop and the angle between the line of sight and the direction of the moving cusp. It can be arbitrarily large; therefore, we take $f_{h}$ to be a free parameter. Here we report on a direct search for these signatures in LIGO and Virgo, and constrain a yet unexplored region of the string parameter space $(G \mu, \varepsilon, p)$.

The search.-The LIGO-Virgo detector network $[35,36]$ is composed of four kilometer-scale Michelson interferometers: $\mathrm{H} 1(4 \mathrm{~km})$ and $\mathrm{H} 2(2 \mathrm{~km})$ share the same location at Hanford, Washington, USA, L1 $(4 \mathrm{~km})$ is in Livingston Parish, Louisiana, USA, and V1 (3 km) is located near Pisa, Italy. We analyze data collected between November 2005 and October 2010, at times when at least two detectors were operating simultaneously in stable conditions. This corresponds to a total of 625 days of observation time.

The search for GW bursts from cosmic strings begins with a matched-filter analysis of strain data from each detector separately [37]. It consists of projecting the whitened data onto an overpopulated [38] template bank defined by a set of cusp waveforms with a high-frequency cutoff spanning from 75 up to $8192 \mathrm{~Hz}$. This procedure results in a time series for the signal-to-noise ratio (SNR) for each template. An event is identified when the SNR $>3.6$ and only the template with the largest SNR is retained when several templates are triggered at the same time. A set of five variables is used to characterize an event. The event time $t_{e}$ and the SNR $\rho$ are determined by the point where the SNR time series is maximum. The triggered template provides the high-frequency cutoff $f_{h}$ and the amplitude $A$. In addition, a $\chi^{2}$ parameter can be computed to characterize the match between the event and the signal waveform in the time domain [39].

Many transient noise events can mimic the properties of a GW burst from a cusp. They constitute the background of our search and reduce our chances of detecting weak signals. A fraction of these events is removed by data quality vetoes specific to each interferometer [40,41]. A stronger handle on background is the requirement of simultaneous detection in two or more interferometers. The central time of the single-detector events must lie within a time window sufficiently large to take into account the maximum light travel time between detectors, the signal duration, and the timing uncertainty. For each pair of detectors, a coincident event is characterized by three 
variables: $\left(\delta t_{e}, r A, r f_{h}\right)$ where $\delta$ is used for the difference and $r$ for the ratio between the two detectors of the pair.

To discriminate true signals from background events, we apply the multivariate technique described in Ref. [42], which uses a set of simulated GW events and typical noise events to statistically infer the probability for a candidate to be signal or noise. Given a set of parameters $\vec{x}$ describing an event $E$, a likelihood ratio can be computed and used to rank the events:

$$
\Lambda(\vec{x})=\frac{P(\mid \vec{x} E \in S)}{P(\vec{x} \mid E \in B)},
$$

where $S$ and $B$ refer to the signal and background training sample, respectively. The background sample is obtained by artificially time shifting the single-detector triggers prior to the coincidence search. The signal sample is generated by injecting simulated cosmic string signals in the detectors' data. The simulated sources are uniformly distributed in volume and the distribution of frequency cutoffs $f_{h}$ is $d N \propto f_{h}^{-5 / 3} d f_{h}$ [37]. The simulated signals are injected on a time-shifted data set in order not to bias the event ranking performed on the nonshifted data.

We parametrize an event by the coincidence variables $\delta t_{e}, r A$, and $r f_{h}$ given for each of the six possible pairs of detectors. These variables allow us to favor signals that are coherent in the network. We also include the single-detector SNR and $\chi^{2}$ parameters to discriminate genuine signals from noise. An additional parameter is the identifier for which set of interferometers was involved in each event, one of 11 possible combinations, to account for the different sensitivity of, for instance, a two-detector network versus a three-detector network. An event is therefore represented by a total of 27 variables. The large dimensionality of the parameter space presents a computational challenge. To obtain statistically reliable results, this method would require very large signal and background samples, well beyond the capabilities of present-day computers. Instead, we assume the parameters to be uncorrelated so the likelihood ratio of Eq. (1) can be factorized as

$$
\Lambda(\vec{x}) \approx \prod_{i=0}^{27} \Lambda\left(x_{i}\right)=\prod_{i=0}^{27} \frac{P\left(x_{i} \mid E \in S\right)}{P\left(x_{i} \mid E \in B\right)} .
$$

This allows us to compute the likelihood ratio one variable at a time. Since this estimator of $\Lambda$ neglects possible correlations between parameters, it might result in the search being less sensitive, compared to the idealized case where the full 27-dimensional likelihood ratio is known. In fact, we do not perform such a factorization for the SNR and $\chi^{2}$ parameters because of the strong correlation between these two variables.

Results.-The LIGO-Virgo data set was divided into 24 time segments, which are analyzed independently. In particular, the training sets $S$ and $B$ are generated for each
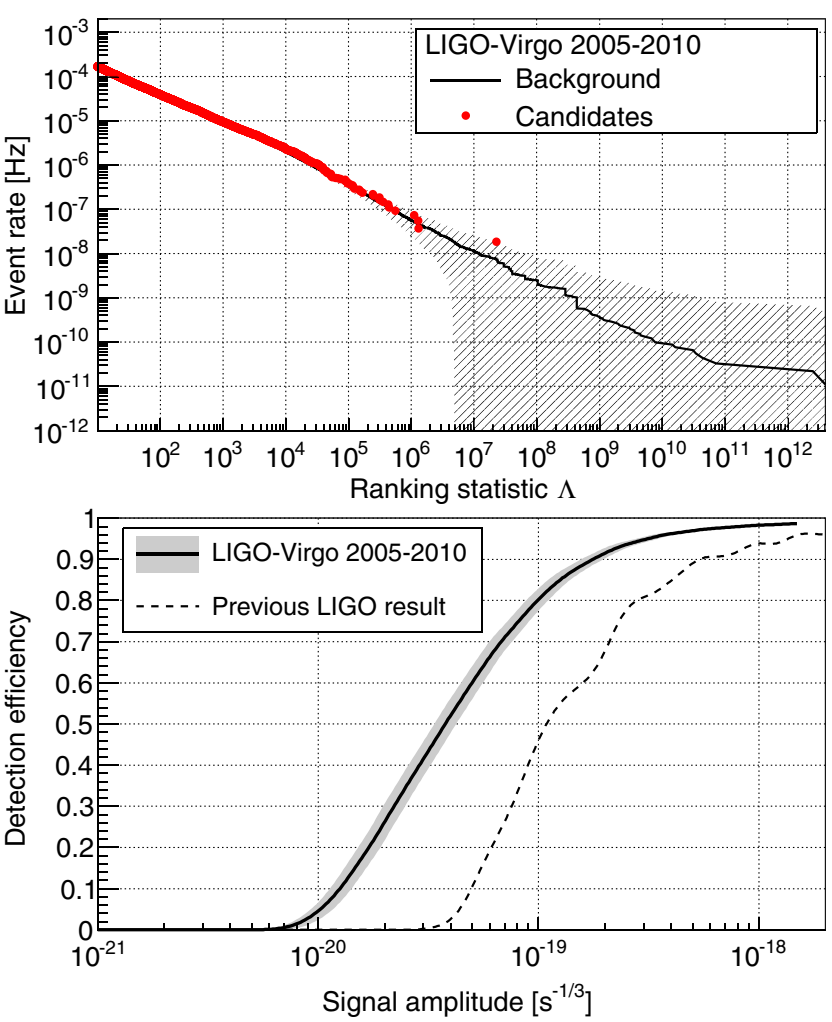

FIG. 1 (color online). In the upper plot, the red circles show the cumulative event rate as a function of the ranking statistic $\Lambda$. The black line shows the expected background of the search with the $1 \sigma$ statistical error represented by the hatched area. The highest-ranked event $\left(\Lambda_{h} \simeq 2.3 \times 10^{7}\right)$ is consistent with the background. The lower plot shows the sensitivity of the search as a function of the signal amplitude. This is measured by the fraction of simulated cusp events recovered with $\Lambda>\Lambda_{h}$. This is to be compared to the sensitivity of the previous LIGO search [32] represented by the dashed line.

segment to account for the noise nonstationarities and the evolution of the detector sensitivities. The principal outcome of this search is shown in Fig. 1. The upper plot in Fig. 1 shows the combined cumulative event rate as a function of the ranking statistic $\Lambda(\vec{x})$. The highest-ranked event of the search occurred on May 10, 2007 at 16:27:15 UTC and is detected simultaneously by the three LIGO interferometers. The ranking value of this event is less than $1 \sigma$ away from the expected background distribution from time-shifted data, shown on the same plot. Therefore, we cannot claim this event to be a GW signal produced by cosmic strings.

To determine the search sensitivity and derive an upper limit, about 7 million simulated cusp signals were injected into a time-shifted data set. To avoid self-selection issues, we use a set of injections that is independent from the $S$ sample used to train the likelihood ranking. We run the search using the same likelihood functions as for the nonshifted analysis and count how many simulated signals are detected with $\Lambda$ larger than the highest-ranked 
event [43]. The lower plot in Fig. 1 shows the detection efficiency $e$ as a function of the injected signal amplitude $A$. The uncertainties associated with the efficiency curve include binomial counting fluctuations, calibration uncertainties, and an amplitude binning uncertainty. This result shows that the search sensitivity has improved by a factor 3 with respect to previous LIGO results [32]. Half of this gain is explained by the increased sensitivity of the detectors; the rest of the gain is due to our improved statistical analysis.

A natural question we wish to answer next is what are the implications of this nondetection for constraints in the cosmic string parameter space. We derived modeldependent upper limits with the method described in Ref. [37] and previously adopted in Ref. [32]. Given the search efficiency $e(A)$, we expect to observe an effective rate of GW bursts given by the integral over the redshift $z$ :

$$
\gamma(G \mu, \varepsilon, p)=\int_{0}^{\infty} e(z ; G \mu, \varepsilon) \frac{d R(z ; G \mu, \varepsilon, p)}{d z} d z,
$$

where $d R(z ; G \mu, \varepsilon, p)$ is the cosmological rate of events with a redshift between $z$ and $z+d z$ and is derived in Ref. [37]. This rate relies on the generic loop density distribution [21]:

$$
n(l, t)=(p \Gamma G \mu)^{-1} t^{-3} \delta(l-\varepsilon \Gamma G \mu t) .
$$

This means that at a given cosmic time, the loop size is given by the gravitational backreaction (the $\delta$ function) and is identical for all loops. Following Ref. [34], the signal amplitude is written as

$$
A(z ; G \mu, \varepsilon)=\frac{g_{1} H_{0}^{1 / 3}(G \mu)^{5 / 3}\left[\varepsilon \Gamma \varphi_{t}(z)\right]^{2 / 3}}{(1+z)^{1 / 3} \varphi_{r}(z)},
$$

where $g_{1}$ is an ignorance constant that absorbs the unknown fraction of the loop length, which contributes to the cusp, and factors of $\mathcal{O}(1) . H_{0}=70.1 \mathrm{~km} \mathrm{~s}^{-1} \mathrm{Mpc}^{-1}$ [44] is the Hubble constant. Two dimensionless cosmologydependent functions of the redshift $z$ enter the amplitude expression: $\varphi_{t}$ and $\varphi_{r}$ relate the redshift and the cosmic time $t=H_{0}^{-1} \varphi_{t}(z)$ and the proper distance $r=H_{0}^{-1} \varphi_{r}(z)$, respectively. We use the $\varphi_{r}$ and $\varphi_{t}$ functions derived in Appendix A of Ref. [37] for a Universe that contains matter and radiation and includes a late-time acceleration. Those functions are computed using the energy densities relative to the critical density: $\Omega_{m}=0.279, \Omega_{r}=8.5 \times 10^{-5}$, and $\Omega_{\Lambda}=0.721$ for the matter, radiation, and cosmological constant, respectively [44].

Knowing how the GW amplitude $A$ scales with redshift [Eq. (5)], the efficiency curve in Fig. 1 can be constructed as a function of the redshift and parametrized with $G \mu$ and $\varepsilon$. As a result, the parameter space $(G \mu, \varepsilon, p)$ can be scanned and the effective rate $\gamma$ computed. The parameter space is ruled out at a $90 \%$ level when the effective rate

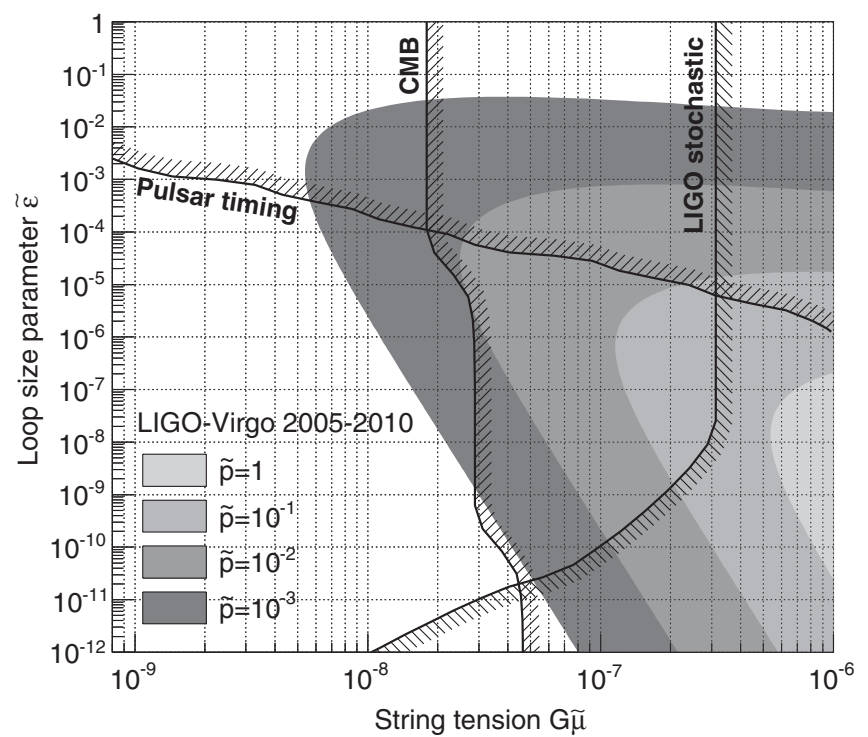

FIG. 2. Constraints on the modified cosmic string parameters $G \tilde{\mu}=g_{1} g_{2}^{-2 / 3} G \mu, \tilde{\varepsilon}=g_{1}^{-1} g_{2}^{5 / 3} \varepsilon$, and $\tilde{p}=\left(n_{c} g_{1}\right)^{-1} g_{2}^{-1 / 3} p$, where $g_{1}, g_{2}$ and $n_{c}$ are numerical factors of $\mathcal{O}(1)$. The gray regions, in different shades for four reconnection probability values, are rejected by our analysis at a $90 \%$ level. The black lines show the bounds derived from the GW stochastic background spectrum for $\tilde{p}=10^{-3}$ and for a small loop scenario (CMB, pulsar, and LIGO data). The rejected region is always on the right-hand side of these lines.

exceeds $2.303 / T$, which is the expected rate from a Poisson process over an observation time $T$. In addition to the ignorance constant $g_{1}$ in Eq. (5), the $d R(z ; G \mu, \varepsilon, p)$ expression given in Ref. [37] includes two other ignorance constants: $g_{2}$, and the average number of cusps per loop oscillation $n_{c}$. These three constants are expected to be of $\mathcal{O}(1)$ provided cosmic string loops are smooth. Instead of fixing these factors to 1 as it is usually done, we choose to absorb these unknown factors in modified cosmic string parameters: $G \tilde{\mu}=g_{1} g_{2}^{-2 / 3} G \mu, \tilde{\varepsilon}=g_{1}^{-1} g_{2}^{5 / 3} \varepsilon$, and $\tilde{p}=\left(n_{c} g_{1}\right)^{-1} g_{2}^{-1 / 3} p$.

Figure 2 displays the region of the cosmic string parameter space that is excluded by our analysis (grayshaded areas). For comparison, we also show limits, fixing $\tilde{p}$ at $10^{-3}$, derived from constraints on the GW stochastic background spectrum. These limits were computed adopting the same cosmic string model and using the same parameters $(G \tilde{\mu}, \tilde{\varepsilon}, \tilde{p})$. Our result improves the indirect CMB bound $[29,30]$ by a factor 3 for intermediate $\tilde{\varepsilon}$ values. It nicely complements existing limits provided by pulsar timing experiments for large $\tilde{\varepsilon}[7,28]$ and by the LIGO stochastic search in the very small loop regime [29].

Conclusion.-We found no evidence for GW bursts produced by cosmic (super)string cusps in LIGO-Virgo data collected between 2005 and 2010. In the absence of a detection, we place significant constraints on cosmic string models, surpassing existing limits from $\mathrm{CMB}$ data. The 
next generation of ground-based GW detectors will probe the cosmic string parameter space further, including, for instance, superstring loops with junctions [45], as the improved sensitivity of Advanced LIGO [41] and Advanced Virgo [46] will allow us to search for cosmic strings with an order of magnitude lower tension.

The authors gratefully acknowledge the support of the United States National Science Foundation for the construction and operation of the LIGO Laboratory, the Science and Technology Facilities Council of the United Kingdom, the Max-Planck-Society, and the State of Niedersachsen/Germany for support of the construction and operation of the GEO600 detector, and the Italian Istituto Nazionale di Fisica Nucleare and the French Centre National de la Recherche Scientifique for the construction and operation of the Virgo detector. The authors also gratefully acknowledge the support of the research by these agencies and by the Australian Research Council, the International Science Linkages program of the Commonwealth of Australia, the Council of Scientific and Industrial Research of India, the Istituto Nazionale di Fisica Nucleare of Italy, the Spanish Ministerio de Economía y Competitividad, the Conselleria d'Economia Hisenda i Innovació of the Govern de les Illes Balears, the Foundation for Fundamental Research on Matter supported by the Netherlands Organisation for Scientific Research, the Polish Ministry of Science and Higher Education, the FOCUS Programme of Foundation for Polish Science, the Royal Society, the Scottish Funding Council, the Scottish Universities Physics Alliance, The National Aeronautics and Space Administration, OTKA of Hungary, the Lyon Institute of Origins (LIO), the National Research Foundation of Korea, Industry Canada and the Province of Ontario through the Ministry of Economic Development and Innovation, the National Science and Engineering Research Council Canada, the Carnegie Trust, the Leverhulme Trust, the David and Lucile Packard Foundation, the Research Corporation, and the Alfred P. Sloan Foundation.

*Deceased.

[1] T. W. B. Kibble, J. Phys. A 9, 1387 (1976).

[2] A. Vilenkin and E. Shellard, Cosmic Strings and Other Topological Defects (Cambridge University Press, Cambridge, England, 1994).

[3] I. B. Zeldovich, Mon. Not. R. Astron. Soc. 192, 663 (1980).

[4] M. Wyman, L. Pogosian, and I. Wasserman, Phys. Rev. D 72, 023513 (2005).

[5] N. Bevis, M. Hindmarsh, M. Kunz, and J. Urrestilla, Phys. Rev. Lett. 100, 021301 (2008).

[6] R. Battye, B. Garbrecht, and A. Moss, Phys. Rev. D 81, 123512 (2010).

[7] R. Battye and A. Moss, Phys. Rev. D 82, 023521 (2010).

[8] P. Ade et al. (Planck Collaboration), arXiv:1303.5085.

[9] A. D. Linde, Phys. Rev. D 49, 748 (1994).
[10] E. J. Copeland, A. R. Liddle, D. H. Lyth, E. D. Stewart, and D. Wands, Phys. Rev. D 49, 6410 (1994).

[11] G. Dvali, Q. Shafi, and R. K. Schaefer, Phys. Rev. Lett. 73, 1886 (1994).

[12] S. Sarangi and S. H. Tye, Phys. Lett. B 536, 185 (2002).

[13] R. Jeannerot, J. Rocher, and M. Sakellariadou, Phys. Rev. D 68, 103514 (2003).

[14] E. Witten, Phys. Lett. B 153, 243 (1985).

[15] E. J. Copeland, L. Pogosian, and T. Vachaspati, Classical Quantum Gravity 28, 204009 (2011).

[16] N. Kaiser and A. Stebbins, Nature (London) 310, 391 (1984).

[17] A. S. Lo and E. L. Wright, arXiv:astro-ph/0503120.

[18] E. Jeong and G. F. Smoot, Astrophys. J. Lett. 661, L1 (2007).

[19] J. Urrestilla, N. Bevis, M. Hindmarsh, and M. Kunz, J. Cosmol. Astropart. Phys. 12 (2011) 021.

[20] J. Dunkley, R. Hlozek, J. Sievers, V. Acquaviva, P. Ade et al., Astrophys. J. 739, 52 (2011).

[21] T. Damour and A. Vilenkin, Phys. Rev. D 71, 063510 (2005).

[22] S. Olmez, V. Mandic, and X. Siemens, Phys. Rev. D 81, 104028 (2010).

[23] M. G. Jackson, N. T. Jones, and J. Polchinski, J. High Energy Phys. 10 (2005) 013.

[24] T. Damour and A. Vilenkin, Phys. Rev. Lett. 85, 3761 (2000).

[25] D. P. Bennett and F. R. Bouchet, Phys. Rev. Lett. 60, 257 (1988).

[26] C. Ringeval, M. Sakellariadou, and F. Bouchet, J. Cosmol. Astropart. Phys. 02 (2007) 023.

[27] J. J. Blanco-Pillado, K. D. Olum, and B. Shlaer, Phys. Rev. D 89, 023512 (2014).

[28] F. A. Jenet, G. B. Hobbs, W. van Straten, R. N. Manchester, M. Bailes, J. P. W. Verbiest, R. T. Edwards, A. W. Hotan, J. M. Sarkissian, and S. M. Ord, Astrophys. J. 653, 1571 (2006).

[29] B. Abbott et al. (LIGO Scientific Collaboration, Virgo Collaboration), Nature (London) 460, 990 (2009).

[30] T. L. Smith, E. Pierpaoli, and M. Kamionkowski, Phys. Rev. Lett. 97, 021301 (2006).

[31] R. H. Cyburt, B. D. Fields, K. A. Olive, and E. Skillman, Astropart. Phys. 23, 313 (2005).

[32] B. Abbott et al. (LIGO Scientific Collaboration), Phys. Rev. D 80, 062002 (2009).

[33] V. Berezinsky, B. Hnatyk, and A. Vilenkin, arXiv:astro-ph/ 0001213

[34] T. Damour and A. Vilenkin, Phys. Rev. D 64, 064008 (2001).

[35] T. Accadia et al. (Virgo Collaboration), JINST 7, P03012 (2012).

[36] B. Abbott et al. (LIGO Scientific Collaboration), Rep. Prog. Phys. 72, 076901 (2009).

[37] X. Siemens, J. Creighton, I. Maor, S. Ray Majumder, K. Cannon, and J. Read, Phys. Rev. D 73, 105001 (2006).

[38] The maximal mismatch between two consecutive templates is 0.001 .

[39] B. Allen, Phys. Rev. D 71, 062001 (2005).

[40] N. Christensen (LIGO and Virgo Scientific Collaborations), Classical Quantum Gravity 27, 194010 (2010). 
[41] J. Aasi et al. (Virgo Collaboration), Classical Quantum Gravity 29, 155002 (2012).

[42] K. C. Cannon, Classical Quantum Gravity 25, 105024 (2008).

[43] P. R. Brady, J. D. Creighton, and A. G. Wiseman, Classical Quantum Gravity 21, S1775 (2004).
[44] http://lambda.gsfc.nasa.gov/product/map/dr3/parameters_ summary.cfm.

[45] P. Binetruy, A. Bohe, T. Hertog, and D. A. Steer, Phys. Rev. D 82, 126007 (2010).

[46] T. Accadia et al., Virgo Document VIR-0128A-12 (2012), https://tds.ego-gw.it/ql/?c=8940. 